\title{
Magnetic Properties of Antiferromagnetic Coupled Co-Pt Stacked Films with Two-Dimensional Array Structures*
}

\author{
Haruki Yamane ${ }^{1}$ and Masanobu Kobayashi ${ }^{2}$ \\ ${ }^{1}$ Akita Industrial Technology Center, Akita 010-1623, Japan \\ ${ }^{2}$ Graduate School of Engineering, Chiba Institute of Technology, Narashino 275-0016, Japan
}

\begin{abstract}
The influence of two-dimensional nano-structures on magnetic properties has been investigated in perpendicular antiferromagnetic coupled (AFC) $\mathrm{Co}_{80} \mathrm{Pt}_{20}$ stacked films. The AFC-samples consisted of [Co-Pt $\left.(10 \mathrm{~nm}) / \mathrm{Ru}(0.46 \mathrm{~nm}) / \mathrm{Co}-\mathrm{Pt}(5 \mathrm{~nm})\right]$ stacked layers, and the magnetization of the 5-nm-thick bottom Co-Pt layers was firstly reversed by AF-coupling. Hexagonal arrays of dots and holes were formed on both (top and bottom) Co-Pt layers and only on the top Co-Pt layer. The interlayer exchange coupling between the top and bottom Co-Pt layers across very thin $\mathrm{Ru}$ interlayer of $0.46 \mathrm{~nm}$ in thickness was kept even after the nanofabrication of about $100 \mathrm{~nm}$ in diameter. The coercivity of dot arrays markedly increased with a decrease in dot diameter, while the magnetic properties of hole arrays were less influenced by the nanoscale patterning. The magnetization rotation of AFC-samples with the patterned top layer changed from multiple to continuous reversal processes with decreasing in patterning size. For the top layer patterned sample, the minor loop shift of the 100 -nm-dot arrays decreased from 240 to $140 \mathrm{kA} / \mathrm{m}$, while the 100-nm-hole arrays showed almost the same strength of interlayer exchange coupling compared with that of continuous film prior to patterning. [doi:10.2320/matertrans.M2014150]
\end{abstract}

(Received April 24, 2014; Accepted June 27, 2014; Published August 8, 2014)

Keywords: magnetic properties, cobalt platinum film, antiferromagnetic coupling, two-dimensional arrays

\section{Introduction}

Magnetic multilayer structures with antiferromagnetic coupling are already used in commercial magnetic devices, such as hard disk heads and magnetic sensors, and are expected to find further use in magnetic recording media and high-capacity magnetic random access memory. ${ }^{1,2)}$ The implementation of multiple states of magnetization arrays in magnetic laminates improves multilevel information storage capacity and magnetic device stability, leading to the miniaturization of various devices. Therefore, the fabrication of nanoscale antiferromagnetic conjugates has recently attracted considerable attention because of the interesting basic physical properties and the potential engineering applications in magnetic devices. ${ }^{3-6)}$

Perpendicularly magnetized films are particularly effective in fabricating highly dense magnetic recordings or the miniaturization of magnetic devices. Various interesting phenomena have been reported in perpendicularly magnetized laminates coupled with antiferromagnetic conjugates via a nonmagnetic interlayer; for example, the fine magnetic domain structure characteristic of antiferromagnetic conjugation, ${ }^{7)}$ the control of the interlayer exchange coupling by controlling the film structure ${ }^{8)}$ and the increase in the magnetic Kerr rotation angle because of the magneto-optical cavity effect. ${ }^{9)}$ Specifically, the magneto-optical effect is expected to improve the performance of devices such as magneto-optical recorders, ${ }^{10)}$ magneto-optical spatial light modulation elements ${ }^{11)}$ and plasmon sensors. ${ }^{12)}$ Reports on photonic crystals or magnetic metal nanostructures presently suggest, for example, that the magneto-optical Faraday effect is enhanced owing to multiple reflections in one-dimensional laminated structures consisting of magnetic oxides ${ }^{13)}$ and the magnetooptical effect is also enhanced owing to plasmon resonance in magnetic thin films of metal nanostructures. ${ }^{14)}$ In contrast, a

*This Paper was Originally Published in Japanese in J. Japan Inst. Met. Mater. 77 (2013) 419-423. two-dimensional periodic structure in an antiferromagnetic coupling laminate with multiple states of magnetization arrays may produce a three-dimensional magneto-photonic crystal and thus further enhance the magneto-optical effect. ${ }^{9}$ )

This study investigates how a two-dimensional nanostructure produced by minute processing, which was added to a perpendicularly magnetized $\mathrm{Co}_{80} \mathrm{Pt}_{20}$ laminate structure coupled with an antiferromagnetic conjugate, influence the magnetic properties. The results are discussed below.

\section{Experimental Methods}

The specimen was produced on a thermally oxidized $\mathrm{Si}$ substrate at room temperature by using the multidimensional magnetron sputtering method. The vacuum did not exceed $7 \times 10^{-6} \mathrm{~Pa}$ and the Ar gas pressure was $0.2 \mathrm{~Pa}$. As shown in Fig. 1(a), the film structure of the antiferromagnetic coupling laminate was $[\mathrm{Co}-\mathrm{Pt}(10 \mathrm{~nm}) / \mathrm{Ru}(\mathrm{x} \mathrm{nm}) / \mathrm{Co}-\mathrm{Pt}(5 \mathrm{~nm}) / \mathrm{Ru}$ $(100 \mathrm{~nm})]$. To produce a Co-Pt alloy film, a composite target that placed the Pt tip on the Co target was used. The number of Pt tip sheets was controlled so that the composition of the Co-Pt film was $80: 20$ at\% based on X-ray photoelectron spectroscopy. The intensity of the exchange coupling acting between the upper and lower Co-Pt ferromagnetic layers was controlled by varying the thickness $\mathrm{x}$ of the $\mathrm{Ru}$ intermediate layer between 0 and $1.5 \mathrm{~nm}$. The Ru protective layer $(2 \mathrm{~nm})$ on the film surface prevents the degradation of magnetic properties owing to surface oxidization during minute processing. In addition, $\mathrm{ZnO}(10 \mathrm{~nm})$ was used as a seed layer to improve the hcp-(001) crystal orientation of the $\mathrm{Ru}$ $(100 \mathrm{~nm})$ ground layer. By applying a magnetic field perpendicular to the film surface, the magnetic properties of the minute continuous film before minute processing was measured with an oscillation-type magnetometer and a spectrum-type Kerr effect measuring apparatus. The crystal structure of the film was evaluated by X-ray diffraction.

Negative resist, electron beam lithography equipment and $\mathrm{Ar}$ ion milling equipment were used in minute processing. 


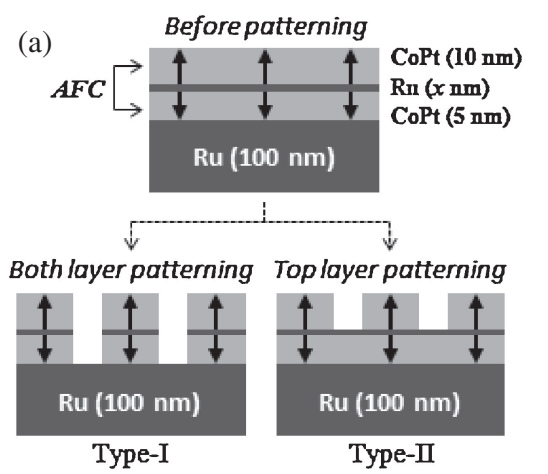

(b)

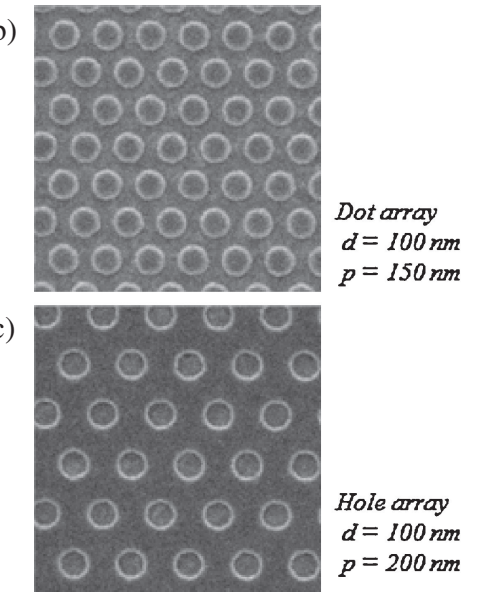

Fig. 1 (a) Schematic illustrations of two types of patterning processes for AFC-samples, and typical SEM images of (b) dot and (c) hole arrays. The interlayer exchange coupling was controlled by the $\mathrm{Ru}$ interlayer thickness $(x)$. The diameter $(d)$ was $100 \mathrm{~nm}$ for the both arrays, and the dot and hole pitches $(p)$ were 150 and $200 \mathrm{~nm}$, respectively.

The conditions for electron beam lithography were $50 \mathrm{kV}$ for accelerating voltage, $50 \mathrm{pA}$ for beam current and $0.8-1.0 \mu \mathrm{s}$ for the exposure time. After developing the resist, pattern processing was performed by etching using Ar ion milling with an accelerating voltage of $0.3 \mathrm{kV}$ and a beam current of $80 \mathrm{~mA}$. Minute processing was performed in an area of $15 \times 15 \mu \mathrm{m}$ that formed hexagonal lattice-shaped dot and hole arrays, and magnetic layers, except in the processing region, which were removed by etching. The diameter $(d)$ of the dots and holes varied from $100 \mathrm{~nm}$ to $15 \mu \mathrm{m}$ and $100 \mathrm{~nm}$ to $1.0 \mu \mathrm{m}$, respectively; furthermore, the ratio of the diameter and space $(\mathrm{p})$ correspondingly varied between $(d: p)=$ $(1.0: 1.5)$ and $(1.0: 2.0)$. Figure 1 (a) shows the four types of minute processing, including a $[\mathrm{Co}-\mathrm{Pt} / \mathrm{Ru} / \mathrm{Co}-\mathrm{Pt}]$ laminate (Type-I), a magnetic film with a $\mathrm{Ru}$ intermediate layer and a single Co-Pt upper film layer (Type-II), each of which had a dot or hole array structure. Scanning electron microscopy (SEM) was used to observe and evaluate the state of minute processing. Figures 1(b) and 1(c) show SEM observations of the (Type-I) specimen. Dot and hole arrays of $100 \mathrm{~nm}$ in diameter were processed on this specimen. The observations show a relatively good two-dimensional periodic structure. The magnetic properties of the as-processed specimen were measured by the pole Kerr effect technique with a maximum magnetic field $875 \mathrm{kA} / \mathrm{m}$ perpendicular to the film surface by converging a semiconductor laser of $405 \mathrm{~nm}$ wavelength onto an approximately $\varphi 5 \mu \mathrm{m}$ area using an object lens.

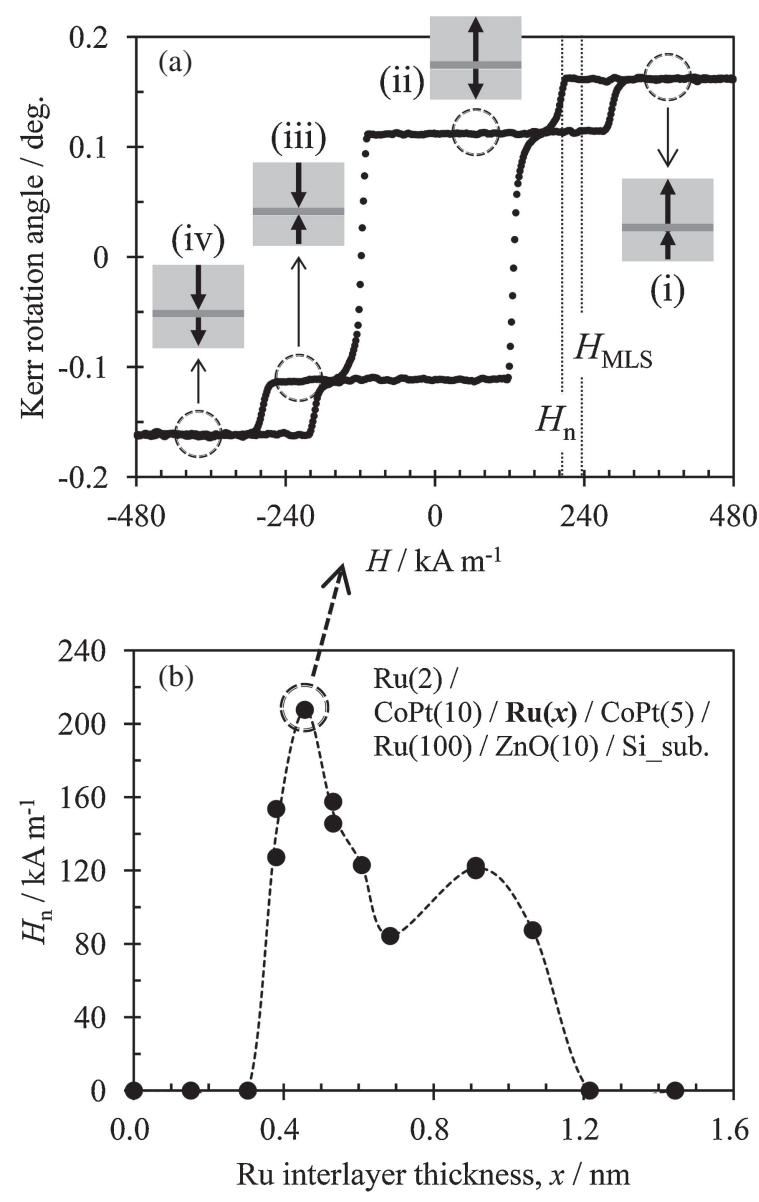

Fig. 2 (a) Magneto-optical (MO) polar Kerr hysteresis loop for an AFCsample with a Ru interlayer of $0.46 \mathrm{~nm}$. (b) Dependence of the Ru interlayer thickness $(x)$ on the magnetic nucleation field $\left(H_{\mathrm{n}}\right)$ of the bottom 5-nm-thick Co-Pt layer.

\section{Experimental Results}

\subsection{Magnetic properties of the antiferromagnetic cou- pling laminate}

Figure 2(a) shows the magnetic Kerr curve perpendicularly to the film and the state of magnetic arrays in the typical Co-Pt antiferromagnetic coupling laminate [Co-Pt $(10 \mathrm{~nm}) /$ $\mathrm{Ru}(0.46 \mathrm{~nm}) / \mathrm{Co}-\mathrm{Pt}(5 \mathrm{~nm}) / \mathrm{Ru}(100 \mathrm{~nm})]$ before minute processing. The step-shaped magnetization curve was accompanied by the magnetic reversal of the upper or lower Co-Pt layer, which suggested relatively good perpendicular magnetic properties and antiferromagnetic coupling. When the applied magnetic field decreased from the upward saturated magnetization state in the upper and lower layers, (i) the magnetization of the lower layer of the Co-Pt $(5 \mathrm{~nm})$ thin film first turns over at approximately $208 \mathrm{kA} / \mathrm{m}$, (ii) the magnetization of the upper layer of the Co-Pt $(10 \mathrm{~nm})$ film turns over at approximately $-110 \mathrm{kA} / \mathrm{m}$ and (iii) the lower layer of the $\mathrm{Co}-\mathrm{Pt}(5 \mathrm{~nm})$ film magnetically reverses upward again owing to antiferromagnetic coupling. Furthermore, by increasing the applied magnetic field in the negative direction, the upper and lower layers fall into a downward magnetized state (iv) and thus the magnetization reversal process is terminated. The intensity of the antiferromagnetic coupling acting between the upper and lower layers through the intermediate $\mathrm{Ru}$ layer is evaluated by the start of the 
reversal of the magnetic field $\left(H_{\mathrm{n}}\right)$ of the Co-Pt $(5 \mathrm{~nm})$ lower film layer, which turns over first. Figure 2(b) shows the $H_{\mathrm{n}}$ dependence on the thickness of the intermediate $\mathrm{Ru}$ layer. Antiferromagnetic coupling is seen in the 0.3-1.2-nm-thick intermediate $\mathrm{Ru}$ layer and is greatest at $0.46 \mathrm{~nm}$ as shown in Fig. 2(a). The state of the vibration of $H_{\mathrm{n}}$ was observed as a function of the Ru-layer thickness. The antiferromagnetic coupling is presumably caused by the RKKY-like exchange interaction between the ferromagnetic layers via the $\mathrm{Ru}$ layer. $\left.{ }^{15}\right)$ In general, the intensity $\left(J_{\mathrm{EX}}\right)$ of the exchange coupling is given by the following formula, which uses the magnetic field shift ( $\left.H_{\mathrm{MLS}}\right)$ that represents the shift from the zero magnetic field of the magnetization curve (minor loop) of the magnetic layer that turns over first. ${ }^{16)}$

$$
J_{\mathrm{EX}}=H_{\mathrm{MLS}} M_{\mathrm{S}} t_{\mathrm{F}}
$$

$M_{\mathrm{S}}$ and $t_{\mathrm{F}}$ are the saturation magnetization and thickness of the magnetic film, respectively. Referring to the antiferromagnetic coupling laminates shown in Fig. 2(a), the magnitude of the exchange coupling of the laminated structure is approximately $1.3 \mathrm{~mJ} / \mathrm{m}^{2}$ because the magnetic field shift of the lower layer of the Co-Pt $(5 \mathrm{~nm})$ film is approximately $240 \mathrm{kA} / \mathrm{m}$.

\subsection{Magnetic properties and ion milling}

The nanoscale thin magnetic film was processed with a high degree of accuracy without degrading the magnetic properties of the laminated structure including the interlayer exchange coupling through the several atoms ultrathin $\mathrm{Ru}$ layer. First, the effect of etching of the magnetic thin film by $\mathrm{Ar}$ ion milling on the magnetic properties of the laminated structure was examined. Figure 3 shows the change in the magnetic properties of the film measured by a spectrum-type Kerr effect measuring apparatus (wavelength $\lambda=405 \mathrm{~nm}$ ) when the entire surface of the Co-Pt antiferromagnetic coupling laminate was etched as shown in Fig. 2(a). The extent of the saturated Kerr rotation angle almost linearly decreases with increasing duration time of etching $\left(t_{\mathrm{E}}\right)$ and thus the Kerr rotation angle drops to nearly zero after etching for $106 \mathrm{~s}$. The upper Co-Pt film thins with etching and the magnetic layer that reverses from the saturated magnetization state owing to the antiferromagnetic coupling moves to the upper layer from the lower layer of the Co-Pt ( $5 \mathrm{~nm})$ film. As shown in Fig. 3, the magnetization curve shift of the upper layer of the Co-Pt $(2 \mathrm{~nm})$ film attributed to the interlayer exchange coupling can be clearly observed even after removing approximately $8 \mathrm{~nm}$ from the upper layer of the Co-Pt film $\left(t_{\mathrm{E}}=60 \mathrm{~s}\right)$. The magnitude of the magnetic field shift is approximately $680 \mathrm{kA} / \mathrm{m}$, and assuming that the saturated magnetization of the upper layer of the Co-Pt film does not decrease with etching, the exchange coupling strength may be maintained at approximately the same strength level as that before etching. Furthermore, the lower layer of the Co-Pt film shows a good square-shaped perpendicular magnetization curve even after the upper layer of the Co-Pt film is carefully removed $\left(t_{\mathrm{E}}=70 \mathrm{~s}\right)$. Thus, the effect of etching the upper film on the perpendicular magnetic properties of the lower film layer is negligible.

The aforementioned results infer that Ar ion milling is an effective processing method that does not significantly affect the magnetic properties of the laminated structure, which

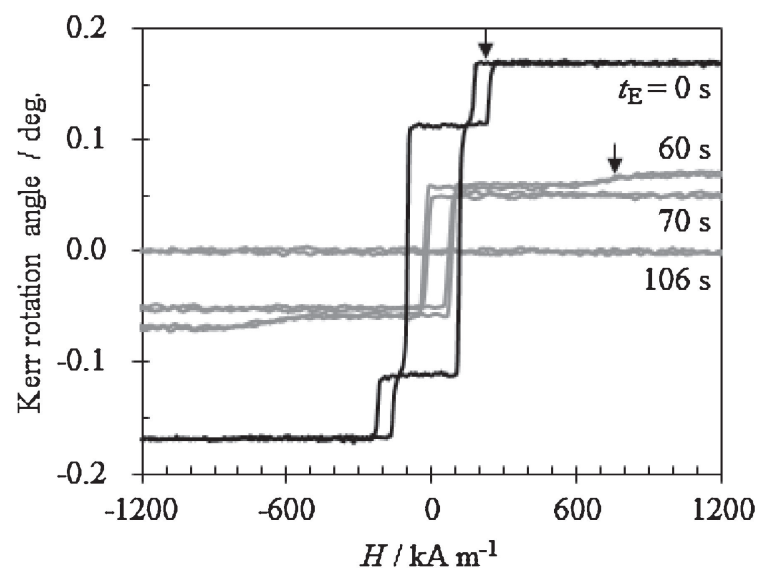

Fig. 3 Influence of Ar ion etching process on magnetic properties for the AFC-sample with the 0.46-nm-thick $\mathrm{Ru}$ interlayer. Two arrows indicate the minor loop shift $\left(H_{\mathrm{MLS}}\right)$ for the AFC-sample before and after etching of $60 \mathrm{~s}$.

includes the interlayer exchange coupling via the ultrathin $(0.46 \mathrm{~nm}) \mathrm{Ru}$ layer.

\subsection{Magnetic properties and the two-dimensional peri- odic structure}

\subsubsection{Minute processing of single-layer Co-Pt film}

The magnetic properties of thin magnetic films are thought to change because of minute processing and the retention force to significantly increase with decreasing dot size. ${ }^{17)}$ To clarify the effect of minute processing on the magnetic properties of thin $\mathrm{Co}-\mathrm{Pt}$ films, dot and hole array processing was carried out on two single-layer Co-Pt films [Co-Pt (5 nm or $10 \mathrm{~nm}) / \mathrm{Ru}(100 \mathrm{~nm})]$ of different thicknesses comprising the antiferromagnetic coupling laminates in Fig. 2(a). Similar to the antiferromagnetic coupling laminate, the $\mathrm{Ru}(2 \mathrm{~nm})$ protective layer is formed on the film surface and $\mathrm{ZnO}$ $(10 \mathrm{~nm})$ is the seed layer on the thermally oxidized $\mathrm{Si}$ substrate. The retention force $\left(H_{\mathrm{c}}\right)$ perpendicular to the film depends on the processing size shown in Fig. 4, and the retention forces of the films greatly increase from tens of $\mathrm{kA} / \mathrm{m}$ to hundreds of $\mathrm{kA} / \mathrm{m}$ with decreasing dot size when the dot array is processed. When the dot diameter is not larger than $1 \mu \mathrm{m}$, the thick single-layer Co-Pt $(10 \mathrm{~nm})$ film shows high retention force. On the other hand, although the retention force slightly increases because of hole array processing, the increase is small compared to the magnetic field shift $\left(H_{\mathrm{MLS}}=240 \mathrm{kA} / \mathrm{m}\right)$ of the lower layer of the CoPt film owing to the antiferromagnetic coupling shown in Fig. 2(a). Therefore, hole array processing appears to have little effect on the antiferromagnetic conjugate. In addition, in hole array processing, the step-shaped magnetization curve changes, suggesting steep magnetic reversal with increasing retention force. As described above, the extra magnetic layer, except in the processing region, is completely removed by etching and similar change is seen in the magnetization curve of the processing region of $15 \times 15 \mu \mathrm{m}$ that does not form holes. Therefore, the specimen region is restricted to $15 \times 15$ $\mu \mathrm{m}$ by minute processing and defects, such as the thick layer distribution and pin holes in the measuring region, decrease and the film quality apparently improves because of the change in magnetic properties due to hole array processing. 


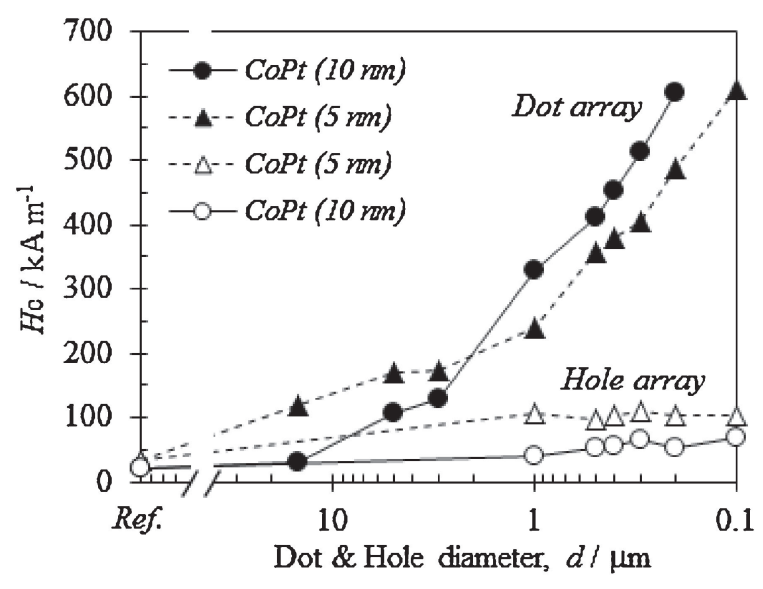

Fig. 4 Dependence of patterning size on coercivity $\left(H_{\mathrm{c}}\right)$ for a 5 -nm and 10 nm-thick Co-Pt single layer films with hexagonal arrays of dots (closed symbol) and holes (open symbol).

\subsubsection{Minute processing and antiferromagnetic coupling laminate}

This study next investigated the effect of two-dimensional periodic structure on the magnetic properties of the $\mathrm{Co}-\mathrm{Pt}$ antiferromagnetic coupling laminate shown in Fig. 2(a). Figure 5 shows the results (Type-I) when dot and hole array processing is performed on the entire magnetic film [Co-Pt $(10 \mathrm{~nm}) / \mathrm{Ru} \quad(0.46 \mathrm{~nm}) / \mathrm{Co}-\mathrm{Pt} \quad(5 \mathrm{~nm})]$ laminate via the intermediate Ru layer. Figure 5(a) shows that the retention force of the upper layer of the Co-Pt film depends on the processing size, and the retention force increases with decreasing processing size in the case of dot arrays, as seen in cases involving single-layer film. In addition, as shown in Figs. 5(b) and 5(d), a significant change was seen in the magnetization curve in the case of dot array processing. As described in the dot processing experiment of the single-layer film, with respect to dots not greater than $1.0 \mu \mathrm{m}$ in diameter, the retention force of the 10-nm-thick Co-Pt film is greater. Therefore, it seems that the magnetization of the lower layer of the $5 \mathrm{~nm} \mathrm{Co-Pt} \mathrm{film} \mathrm{turns} \mathrm{over} \mathrm{first} \mathrm{in} \mathrm{the} \mathrm{laminated}$ structure. The steep magnetic reversal of the lower layer of the Co-Pt film is not seen in dot processing of $1.0 \mu \mathrm{m}$ in diameter. Furthermore, the distribution of the magnetic reversal field of the upper and lower layers increases in the case of dots $100 \mathrm{~nm}$ in diameter.

In contrast, minute processing apparently has little effect on the magnetic properties of hole arrays and the antiferromagnetic coupling via the several atoms ultrathin $\mathrm{Ru}$ layer is maintained at the same prior level after minute processing of $100 \mathrm{~nm}$ as shown in Fig. 5(e). Compared to the continuous film in Fig. 2(a), the magnetic field shift of the lower film layer and the retention force of the upper film layer slightly increase. The increase is attributed to the improved film quality within the measurement region of the narrow specimen region as in the case of the single-layer film.

Regarding the minute processing (Type-II) of the upper layer of the $10 \mathrm{~nm} \mathrm{Co-Pt} \mathrm{film,} \mathrm{the} \mathrm{dependence} \mathrm{on} \mathrm{the}$ processing size is seen in the hole and dot arrays for the magnetic reversal process shown in Fig. 6. In holes and dots of $1.0 \mu \mathrm{m}$ diameter, the magnetization curve suggests that the Co-Pt $(5 \mathrm{~nm})$ lower film layer should follow two steps of magnetic reversal. This can be explained by assuming that the
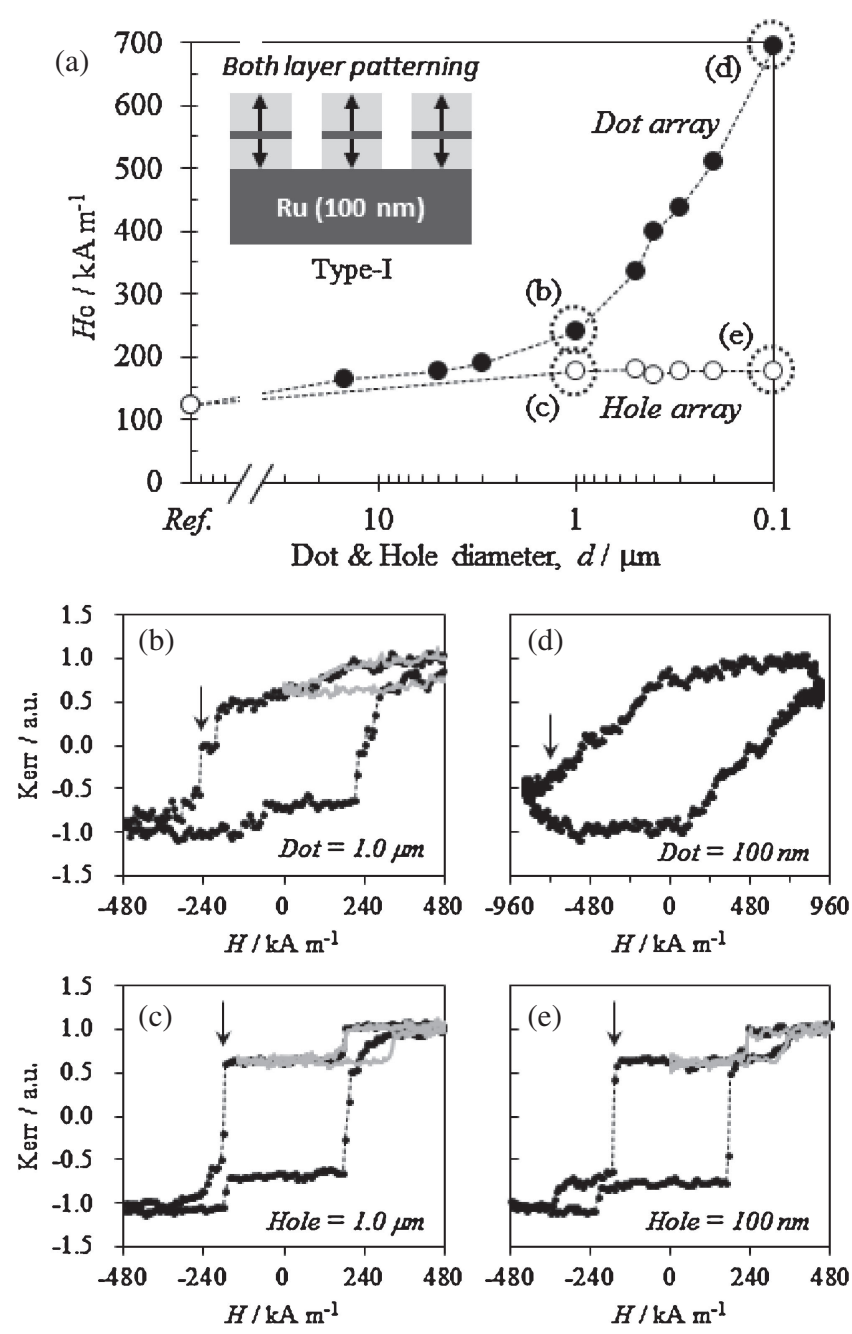

Fig. 5 (a) Dependence of dot (closed symbol) and hole (open symbol) diameters $(d)$ on the coercivity $\left(H_{\mathrm{c}}\right)$ of the top Co-Pt layer for a both layer patterned AFC-sample. MO loops for dot arrays of (b) $1.0 \mu \mathrm{m}$ and (d) $100 \mathrm{~nm}$ in diameter, and for hole arrays of (c) $1.0 \mu \mathrm{m}$ and (e) $100 \mathrm{~nm}$ in diameter. Gray lines show the minor loops for the bottom Co-Pt layer, and the arrows in (b), (c), (d) and (e) indicate the coercivity of the top Co-Pt layer.

Co-Pt lower film layer on the top of which is the upper film layer is first magnetically reversed by antiferromagnetic coupling and then the rest of the lower film layer on the top of which there is no upper layer film left is magnetically reversed. Furthermore, when looking at the magnetization curve of the lower film layer on the top of which there is no upper film layer left, the shift in the magnetization curve is not seen in the case of dot arrays, whereas a magnetic field shift of approximately $120 \mathrm{kA} / \mathrm{m}$ is identified in the hole arrays. These two magnetic reversal steps of the lower film layer disappear with decreases in the processing size of the upper film layer; furthermore, when the diameter of the holes and dots is $100 \mathrm{~nm}$, the entire lower film changes into a single magnetic reversal. In particular, for the specimen processed with hole arrays, the magnetic field shift $\left(H_{\mathrm{MLS}}=250 \mathrm{kA} / \mathrm{m}\right)$ of the lower Co-Pt film layer is at the same level as in the case of the continuous film before minute processing, although the upper Co-Pt film layer decreased because of etching. For the specimen processed with dot arrays, the retention force of the upper film layer increased with decreasing dot size; furthermore, for $100-\mathrm{nm}$ holes and dots, the retention force 

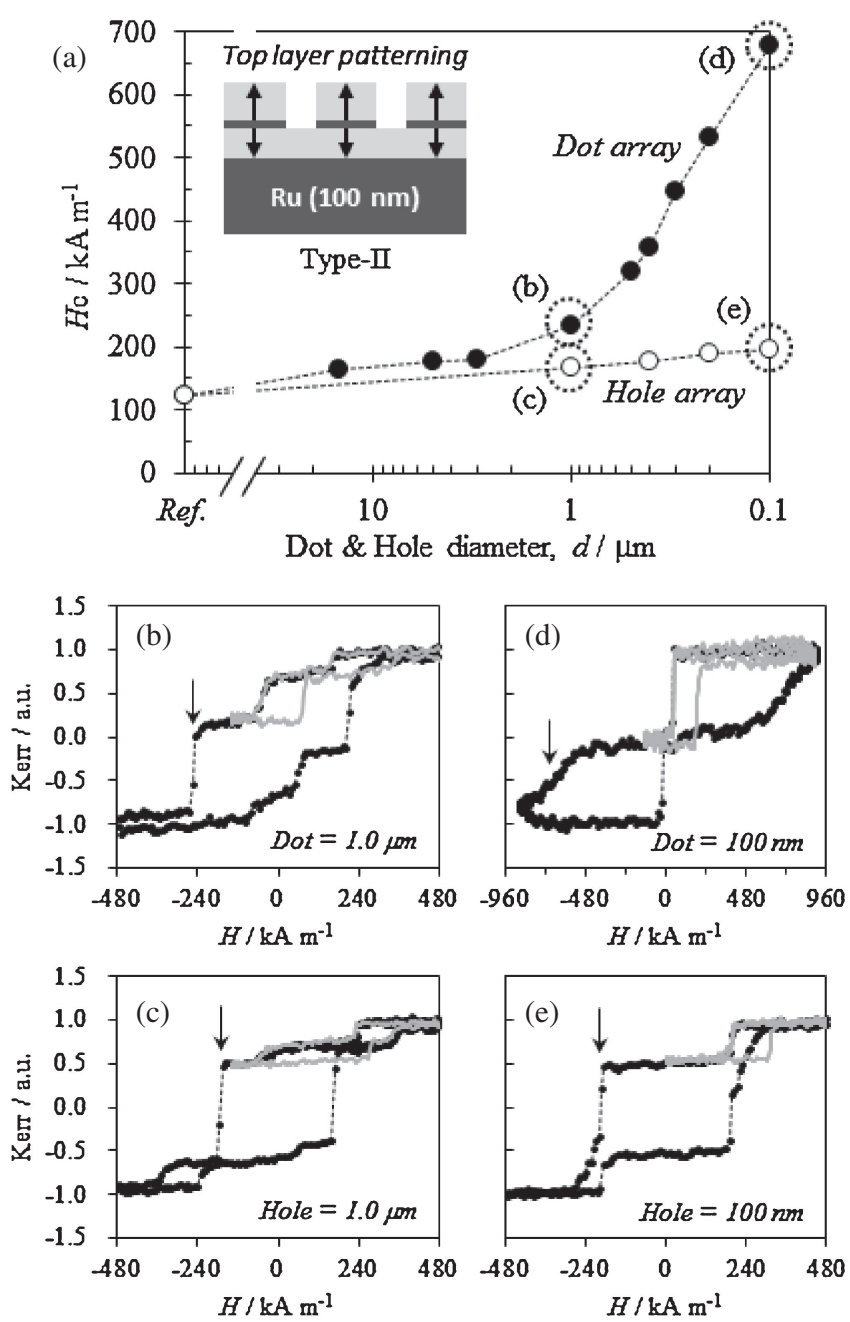

Fig. 6 (a) Dependence of dot (closed symbol) and hole (open symbol) diameters $(d)$ on the coercivity $\left(H_{\mathrm{c}}\right)$ of the top Co-Pt layer for a top layer patterned AFC-sample. MO loops for dot arrays of (b) $1.0 \mu \mathrm{m}$ and (d) $100 \mathrm{~nm}$ in diameter, and for hole arrays of (c) $1.0 \mu \mathrm{m}$ and (e) $100 \mathrm{~nm}$ in diameter. Gray lines show the minor loops for the bottom Co-Pt layer, and the arrows in (b), (c), (d) and (e) indicate the coercivity of the top Co-Pt layer.

increased to approximately $680 \mathrm{kA} / \mathrm{m}$. The magnetic field shift of the lower Co-Pt film layer decreased to approximately $140 \mathrm{kA} / \mathrm{m}$, whereas the entire lower film layer including the part of the film on which dots did not form resulted in magnetic reversal with decreasing distance between the dots. As a result, the magnetization curve, which significantly differs from that of the results in Fig. 5(d) and in which dot arrays are processed on the entire film layer, can be identified.

\section{Conclusion}

The effect of two-dimensional periodic structure on the magnetic properties of a perpendicularly magnetized $\mathrm{Co}-\mathrm{Pt}$ laminate with antiferromagnetic coupling was experimentally studied. To study the effect of minute processing on the antiferromagnetic coupling laminate, dot and hole array processing was performed on [Co-Pt $(10 \mathrm{~nm}) / \mathrm{Ru}(0.46 \mathrm{~nm}) /$ Co-Pt $(5 \mathrm{~nm})]$ film comprising the entire magnetic layer or only the upper $10 \mathrm{~nm} \mathrm{Co-Pt} \mathrm{film} \mathrm{layer.}$

In minute processing by electron beam lithography and $\mathrm{Ar}$ ion milling, the interlayer exchange coupling via an ultrathin
$\mathrm{Ru}$ layer of $0.46 \mathrm{~nm}$ was maintained after processing about $100 \mathrm{~nm}$. In the case of dot arrays, for minute processing of the entire $[\mathrm{Co}-\mathrm{Pt} / \mathrm{Ru} / \mathrm{Co}-\mathrm{Pt}]$ film layer comprising the antiferromagnetic conjugate, the retention force increased with decreasing processing size. In contrast, in the case of hole arrays, the retention force did not much depend on the processing size. Thus, the exchange coupling was almost the same as that before minute processing. On the other hand, in the case of minute processing of the upper film layer, the dependence on the processing size of the magnetic reversal process was observed in the dot and hole arrays. When the diameter was $200 \mathrm{~nm}$ or more, a multistage magnetization curve with partial magnetic reversal of the lower film was observed regardless of the presence or absence of the upper film layer. In contrast, in the case of the $100 \mathrm{~nm}$ diameter, the entire lower film layer went through a magnetic reversal at once. At this point, the magnetic field shift of the lower film layer was at the same level as in the case of the continuous film before minute processing despite the fact that the upper film layer decreased because of etching.

\section{Acknowledgement}

This study was partially supported by the Ministry of Education, Culture, Sports, Science and Technology, Grantin-Aid for Scientific Research, Basic Research Program (C) 23560429 .

\section{REFERENCES}

1) S. Mangin, D. Ravelosona, J. A. Katine, M. J. Carey, B. D. Terris and E. E. Fullerton: Nature Mater. 5 (2006) 210-215.

2) D. A. Allwood, G. Xiong, C. C. Faulkner, D. Atkinson, D. Petit and R. P. Cowburn: Science 309 (2005) 1688-1692.

3) S. N. Piramanayagam, K. O. Aung, S. Deng and R. Sbiaa: J. Appl. Phys. 105 (2009) 07C118.

4) M. V. Lubarda, S. Li, B. Livshitz, E. E. Fullerton and V. Lomakin: Appl. Phys. Lett. 98 (2011) 012513.

5) D. Tripathy, A. O. Adeyeye and N. Singh: Appl. Phys. Lett. 93 (2008) 022502.

6) M. Ranjbar, S. N. Piramanayagam, R. Sbiaa and T. C. Chong: J. Appl. Phys. 111 (2012) 07B921.

7) Y. Fu, W. Pei, J. Yuan, T. Wang, T. Hasegawa, T. Washiya, H. Saito and S. Ishio: Appl. Phys. Lett. 91 (2007) 152505.

8) Y. Fu, S. Ishio, T. Wang, W. Pei, T. Hasegawa, H. Yamane and H. Saito: J. Appl. Phys. 105 (2009) 07C307.

9) H. Yamane: Jpn. J. Appl. Phys. 51 (2012) 095802.

10) R. J. Gambino and T. Suzuki: Magneto-Optical Recording Materials, (Wiley-IEEE Press, 1999).

11) K. Aoshima, N. Funabashi, K. Machida, Y. Miyamoto, N. Kawamura, K. Kuga, N. Shimidzu and F. Sato: Appl. Phys. Lett. 91 (2007) 052507.

12) D. Regatos, D. Farina, A. Calle, A. Cebollada, B. Sepulveda, G. Armelles and L. M. Lechuga: J. Appl. Phys. 108 (2010) 054502.

13) M. Inoue, R. Fujikawa, A. Baryshev, A. Khanikaev, P. B. Lim, H. Uchida, O. Aktsipetrov, A. Fedyanin, T. Murzina and A. Granovsky: J. Phys. D 39 (2006) R151.

14) S. Tomita, T. Kato, S. Tsunashima, S. Iwata, M. Fujii and S. Hayashi: Phys. Rev. Lett. 96 (2006) 167402.

15) S. S. P. Parkin, N. More and K. P. Roche: Phys. Rev. Lett. 64 (1990) 2304-2307.

16) O. Hellwig, A. Berger and E. E. Fullerton: Phys. Rev. Lett. 91 (2003) 197203.

17) C. A. Ross, H. I. Smith, T. Savas, M. Schattenburg, M. Farhoud, M. Hwang, M. Walsh, M. C. Abraham and R. J. Ram: J. Vac. Sci. Technol. B 17 (1999) 3168-3176. 